\title{
NEON ION BOMBARDMENT ON SILICON SURFACES *
}

\author{
A.H.M. HOLTSLAG and A. VAN SILFHOUT \\ Department of Applied Physics, Twente University of Technology, P.O. Box 217, 7500 AE Enschede. The Netherlands
}

\begin{abstract}
Under UHV conditions, c-Si (111), (110), (100) surfaces have been bombarded at room temperature and normal incidence, by $\mathrm{Ne}^{+}$ions. Using spectroscopic ellipsometry $(1.5-5.5 \mathrm{eV})$, the implantation processes were continuously recorded. The results of the optical measurements are compared with the relation: $\left(\epsilon_{2} E^{p}\right)^{q}=c\left(E-E_{0}\right)$, in which Tauc takes $p=2, q=1 / 2$, Davis and Mott $p=2, q=1 / 3$ and Cody $p=0, q=1 / 2$, for $1.5-3.0 \mathrm{eV}$. Applying the least-squares method to the results of the ellipsometric measurements we find that they can be described most satisfactorily by using the model of Davis and Mott. The thickness of the amorphous silicon layer, both during and at the end of bombardment, is calculated from the same optical measurements and compared with the predicted ion range. The growth of the amorphous layer can be described by a simple mathematical model (microscopic surface roughness). On termination of the bombardment we observe a partial restoration of the amorphous layer at room temperature, which may be easily interpreted in terms of the Davis and Mott model.
\end{abstract}

\section{Introduction-experimental}

During ion bombardment on clean c-Si, the various effects induced at the surface (surface roughness, surface-state changes, surface-anisotropy changes) and in the implantation layer (damage, implanted ions, dilatation) are reflected in changes of the complex dielectric function $\tilde{\epsilon}=\epsilon_{1}-i \epsilon_{2}$ and the measurable ellipsometric quantities $\Psi$ and $\Delta\left(\tilde{\rho}=\tan \Psi \mathrm{e}^{\mathrm{i} \Delta}\right)$. The damage to the layer will be a low-dose effect. Measurable effects of implanted ions, dilatation and/or surface roughness arising during bombardment will be a high-dose effect.

In order to distinguish between the different effects mentioned, two ellipsometric studies were carried out, which will be partially reported:

a) Clean c-Si (111), (110), (100) substrates, were bombarded with $\mathrm{Ne}^{+}$ions having an energy of $2 \mathrm{keV}$. During bombardment $\Psi$ and $\Delta$ were measured at four wavelengths in the ultraviolet photon-energy range. In this case the intensity penetration depth of the light for some of the photon energies is less than the layer thickness and the (psuedo-)dielectric constant of the layer can be obtained directly; it appears as if the measurements were made on a varying substrate with infinite thickness.

b) Clean c-Si (111) surfaces were bombarded with $\mathrm{Ne}^{+}$ions having different acceleration voltages (0.5-2.5

\footnotetext{
* This work is part of the research program of the "Stichting voor Fundamenteel Onderzoek der Materie" (Foundation for Fundamental Research on Matter) and was made possible by financial support from the "Nederlandse Organisatie voor Wetenschappelijk Onderzoek" (Netherlands Organisation for the Advancement of Pure Research).
}

$\mathrm{kV}$ ). During an increasing dose, $\Psi$ and $\Delta$ were measured at four wavelengths in the visible photon-energy range. In this case the intensity penetration depth of the light is greater than the layer thickness and a layer thickness as a function of the acceleration voltage can be obtained. The $\Psi$ and $\Delta$ values were measured over the whole photon-energy range $(1.5-5.5 \mathrm{eV})$ before and after bombardment.

The experiments were carried out in a UHV system (base pressure $10^{-8} \mathrm{~Pa}$ ), having facilities for sampletemperature control, spectroscopic PSA rot ellipsometry, AES, mass-selective ion bombardment, sputtering and mass spectrometry [1]. Before bombardment the silicon samples were cleaned by argon ion sputtering $\left(45^{\circ}\right.$, $800 \mathrm{eV}, 2 \times 10^{-6} \mathrm{~A} \mathrm{~cm}^{-2}$ ) followed by an anneal for one hour at $800^{\circ} \mathrm{C}$ and checked by AES. The massselective ion bombardments at normal incidence with a flux of $115 \times 10^{-9} \mathrm{Acm}^{-2}$ were carried out at room temperature in all cases $\left(T_{0}=28^{\circ} \mathrm{C}\right)$.

\section{Results}

In order to obtain an idea of the events during bombardment, the pseudodielectric constants of the two highest photon-energies which have been used during bombardment, are plotted in the $\left(\epsilon_{1}, \epsilon_{2}\right)$ plane, see fig. 1. Firstly the curves bend from the starting points at $\tilde{\epsilon}_{c}$ (crystalline) of c-Si in different directions, because $\tilde{\epsilon}_{\mathrm{c}}$ and $\tilde{\epsilon}_{\mathrm{a}}$ (amorphous) are functions of the photon energy, but they are finally directed to the point $\tilde{\epsilon}_{\mathrm{v}}=\left(\epsilon_{1}, \epsilon_{2}\right)=$ $(1,0)$. This shows that at a high ion dose a dilution is obtained. Similar results are observed for bombardments with $\mathrm{Ar}^{+}$ions [1]. At an increasing dose $N_{\mathrm{b}}$ 


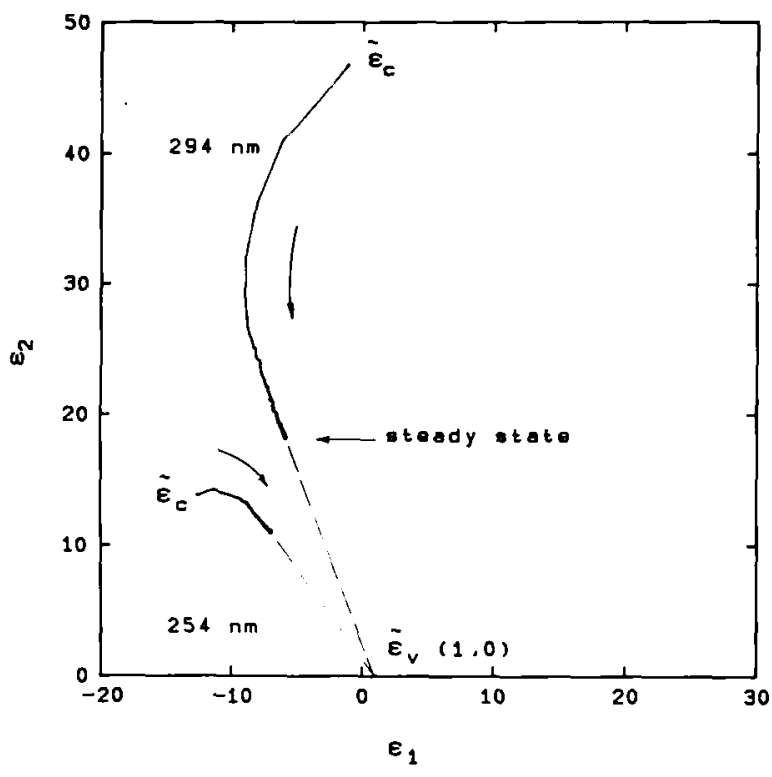

Fig. 1. The pseudodielectric constants at a high-photonenergy during $2 \mathrm{keV} \mathrm{Ne}{ }^{+}$ion bombardment on $\mathrm{c}-\mathrm{Si}$ (111) presented in the $\left(\epsilon_{1}, \epsilon_{2}\right)$ plane. The arrows denote the direction due to an increasing ion dose.
$\left(\AA^{-2}\right)$. The low ion-dose effect can be described by an exponential function, $\theta_{\mathrm{c}}=\exp \left(-N_{\mathrm{b}} A_{\mathrm{a}}\right)$ with $A_{\mathrm{a}}=$ $18 \AA^{2}$ at $U_{\mathrm{v}}=2 \mathrm{kV}$ for all three surfaces and confirms the predicted behaviour of the model of Morehead and Crowder [2], see also fig. 4.

We shall now only discuss measurements in the visible photon-energy range $(1.5-3 \mathrm{eV})$. If the bombardment is terminated, the values of $\delta \Psi$ and $\delta \Delta$ still change, which can be explained by damage recovery at room temperature (self-anneal behaviour). Therefore we bombarded until the steady state was reached and then made a scan over the whole photon-energy range. After this procedure the bombardment was terminated and the optical data were again measured after $1 \mathrm{~h}$. By means of this procedure the effects can be treated separately. Fig. 2 contains the ellipsometric data for an ion dose of $N_{\mathrm{b}}=1.7 \AA^{-2}$ after the self-anneal behaviour. Also the data at a dose of $N_{\mathrm{b}}=4.2 \AA^{-2}$ during the steady state and after the self-anneal behaviour, as a function of photon energy are included. Both ion doses are beyond the low-dose effect, therefore the curves look much the same. In order to describe the results we shall first assume that the implanted layer can be regarded as homogeneous with a certain effective dielectric constant

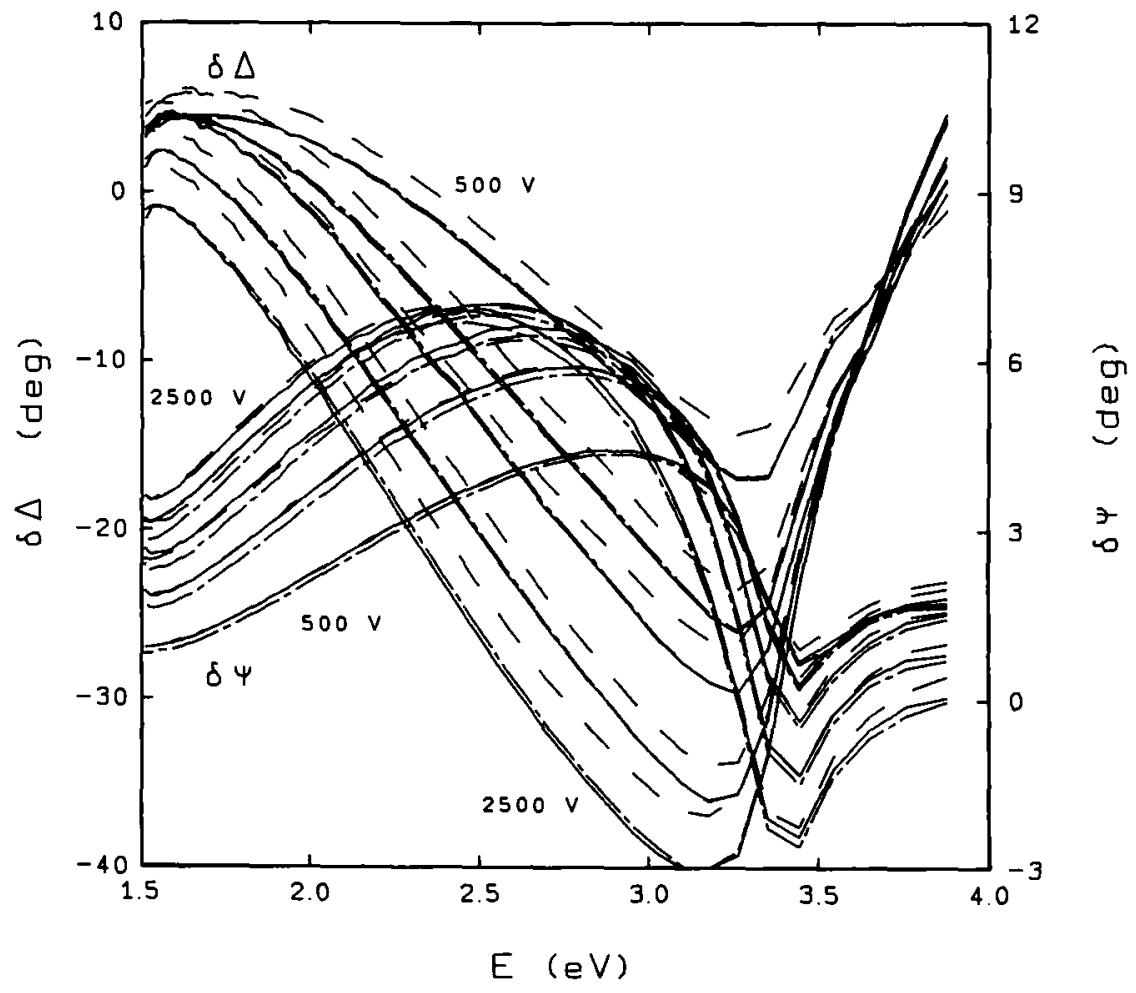

Fig. 2. The ellipsometric values after an ion dose of $1.7 \AA^{-2}(--)$ and $4.2 \AA^{-2}(---)$ recorded one hour after termination of the bombardment. Also included are the steady-state values at a dose of $\approx 4.2 \AA^{-2}(-)\left(\phi_{0}=69.9^{\circ}\right)$. The acceleration voltage is indicated. 
$\tilde{\epsilon}_{l}$ and layer thickness $d$, on top of a crystalline substrate. Furthermore $\tilde{\boldsymbol{\epsilon}}_{\text {, }}$ can be specified for instance with the effective medium approximation as soon as the composition of the layer is known.

\section{Calculations}

After a photon-energy scan, the values of $\tilde{\rho}$ at $M$ different photon energies are known and are called $\tilde{\rho}_{m}$ at a photon energy $E_{m}$. We have to fulfill $2 M$ equations, with $M$ unknown pairs $\left(\epsilon_{1 l}, \epsilon_{2 l}\right)$ and an unknown layer thickness $d$, so that we have $2 M+1$ unknown values:

$$
\begin{array}{r}
\tilde{\rho}_{l}\left(\tilde{\epsilon}_{l}\left(E_{m}\right), d, \text { known parameters }\right)-\tilde{\rho}_{\mathrm{m}}=0+\mathrm{i} 0 \\
1 \leqq m \leqq M .
\end{array}
$$

If we choose $d$ it is possible to determine all pairs $\left(\epsilon_{1 l}, \epsilon_{2 l}\right)$, by applying the Newton-Raphson method for instance. We will take an extra relation for $\epsilon_{2 l}(E)$ as a function of the photon energy $E$. The layer thickness $d$ where $\epsilon_{2 /}(E)$ obeys the relation, will be considered [1] as the correct layer thickness.

In the low photon-energy range $1.5 \mathrm{eV} \leqq E \leqq 3.0 \mathrm{eV}$, the imaginary part of the dielectric constant $\epsilon_{2 a}$ of amorphous silicon is assumed to obey the relation: $\left(\epsilon_{2 \mathrm{a}} E^{p}\right)^{q}=c\left(E-E_{\mathrm{g} 0}\right)$, where $E_{\mathrm{g} 0}$ denotes an optical bandgap, $c$ is a constant and $p$ and $q$ are values dependent on the choice of the author [3-5]. Also in the case that a dilution arises this formula can be used. From an approximation [1] of the EMA equation for a low void fraction $\theta_{\mathrm{v}}$ it follows that $\tilde{\epsilon}_{l}=\tilde{\epsilon}_{\mathrm{a}}\left(1-3 / 2 \theta_{\mathrm{v}}\right)$, thus:

$\left(\epsilon_{2 l} E^{p}\right)^{q}=\left(1-\frac{3}{2} \theta_{\mathrm{v}}\right)^{q}\left(\epsilon_{2 \mathrm{a}} E^{p}\right)^{q}=c_{\mathrm{v}}\left(E-E_{\mathrm{g} 0}\right)$,

with a new value $c_{v}=c\left(1-3 / 2 \theta_{v}\right)^{q}$, from which the void fraction can be obtained. By making use of a linear regression technique [1] the next results are obtained. Fig. 3 is a plot of the $\left(\epsilon_{2 l} E^{p}\right)^{q}$ data obtained as a function of the photon energy. Thus, in the photonenergy range mentioned, Tauc's [3] formula with $p=2$ and $q=1 / 2\left(E_{\mathrm{g} 0}=1.30 \mathrm{eV}\right)$ does not give a straight line at all, but the model of Davis and Mott [4] where $p=2$ and $q=1 / 3\left(E_{\mathrm{g} 0}=0.92 \mathrm{eV}\right)$ and that of Cody et al. [5] where $p=0$ and $q=1 / 2\left(E_{80}=0.85 \mathrm{eV}\right)$ do show straight lines. However the model of Davis and Mott give for different acceleration voltages, respectively different layer thicknesses, a smaller deviation in the $\epsilon_{21}$ values [1]. The data are obtained from the measured $\delta \Psi$ and $\delta \Delta$ values in the steady state.

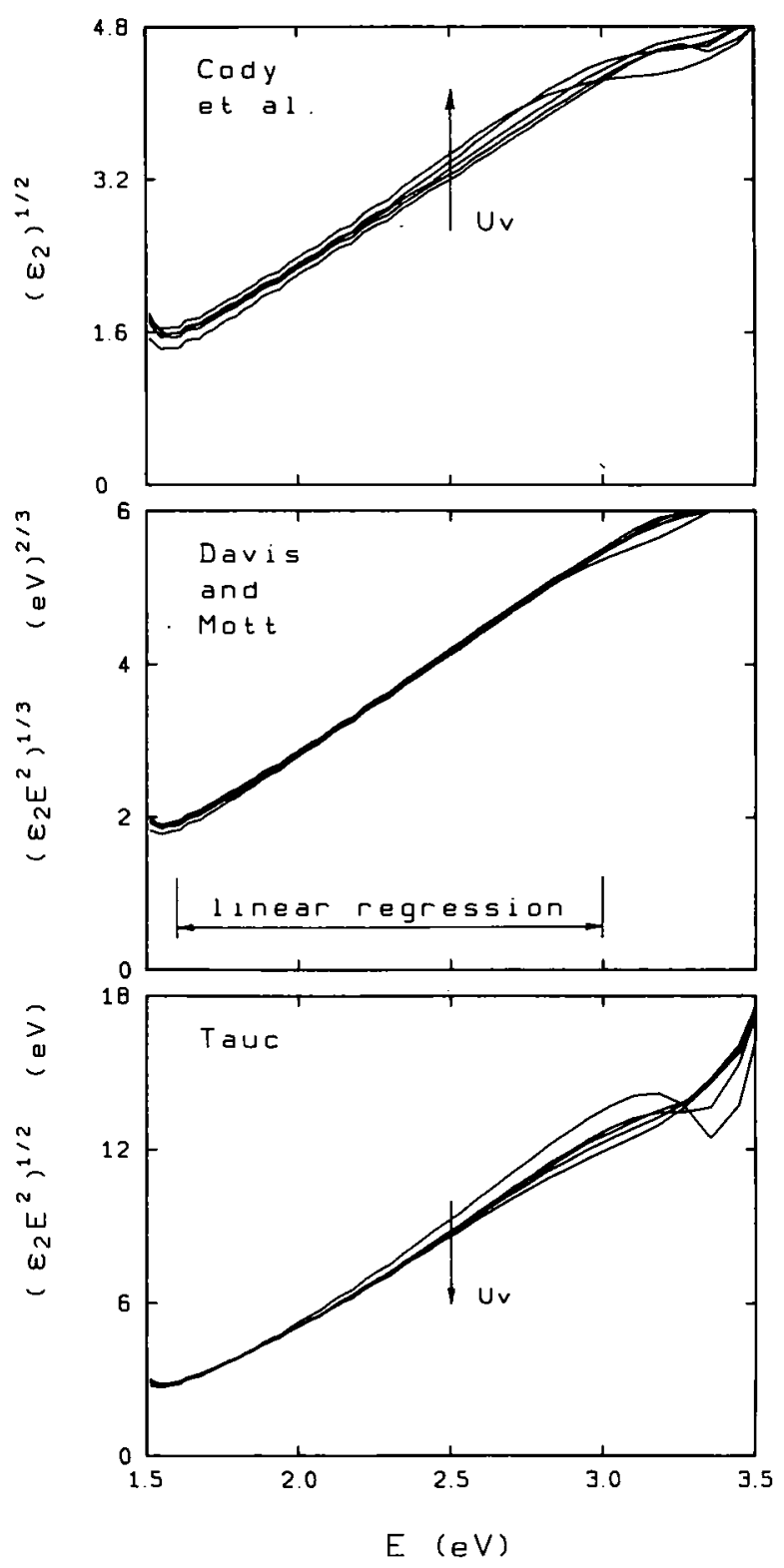

Fig. 3. A comparison of the relations $\left(\epsilon_{2} E^{p}\right)^{q}$ as a function of the photon energy, at different acceleration voltages $U_{v}$.

\section{Discussion}

\subsection{Anneal behaviour at room temperature}

In the case of the $\delta \Psi$ and $\delta \Delta$ measurements, recorded one hour after termination of the bombardment, the values $c, E_{g 0}$ and the layer thickness $d$ are also calculated. It turns out that the calculated layer thickness remains the same (within $0.1 \AA$ ) as in the steady state. The same layer thickness means that the interface $\mathrm{a}-\mathrm{Si} / \mathrm{c}-\mathrm{Si}$ remains fixed in space at room temperature 
after termination of the bombardment. Furthermore $E_{\mathrm{g} 0}$ increases for all corresponding measurements by $0.04 \mathrm{eV}$, even while a certain deviation in the $E_{\mathrm{g} 0}$ values for different experiments is present. Within the scope of the model of Davis and Mott, an increasing $E_{\mathrm{g} 0}$ means a lower density of states in the original crystalline gap after self-anneal. We have to be careful with the interpretation of this effect, because it can also be a surface recovery. However we have looked in detail into this effect [1] by again applying the same bombardment after the self-anneal effect but now as a function of the sample temperature. From the measured relaxation times an Arrhenius plot is obtained from which an activation energy of $\approx 64 \mathrm{meV}$ is derived. This value corresponds to a fitting parameter in the model of Morehead and Crowder [2] which explains the temperature dependence of area $A_{\mathrm{a}}$.

\subsection{Ranges}

The ion range $r$, projected range $x_{\mathrm{p}}$, range stragglings $\sigma_{x}$ and $\sigma_{z}$ are calculated [1] from Biersack's model [6]. The density of the target $n_{\mathrm{si}}=0.05 \AA^{-3}$ is taken as constant and the implanted noble gas is neglected. These values can be used to explain the low-dose

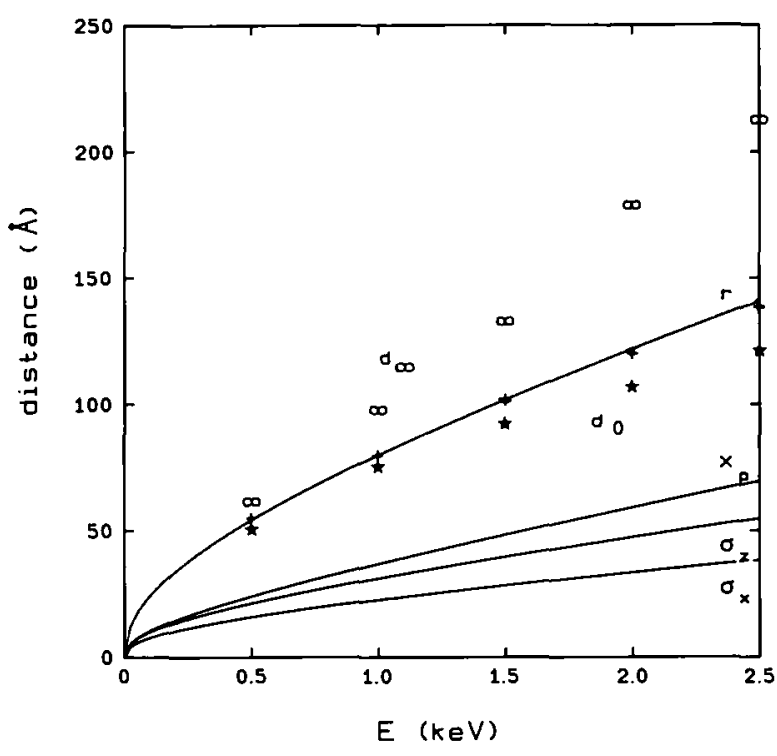

Fig. 5. A comparison between observed and calculated layer thicknesses. The layer thickness at $N_{\mathrm{b}}=0.20 \AA^{-2}(+)$ where $\theta_{c}=0$ corresponds with the ion range $r$. Also the observed values $d_{0}(*)$ and $d_{\infty}(\infty)$ are included. The values of the projected range $x_{\mathrm{p}}$, range stragglings $\sigma_{x}$ and $\sigma_{z}$ are calculated from Biersack's model [6].

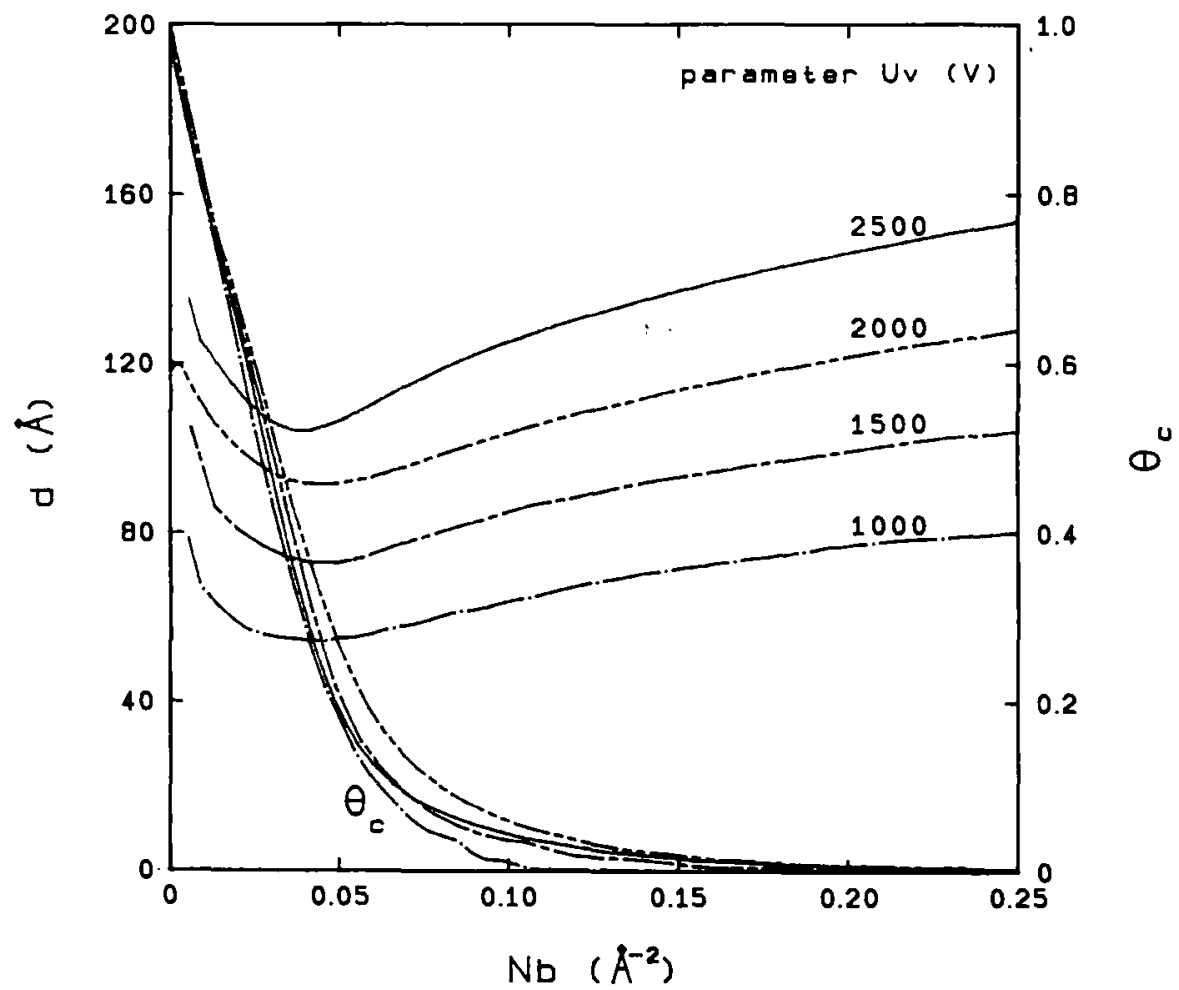

Fig. 4. The layer thickness $d$ and crystalline fraction $\theta_{\mathrm{c}}$ as a function of the ion dose $N_{\mathrm{b}}$ during the low-dose behaviour. 
behaviour, neglecting channeling, see fig. 4 . The layer thicknesses at $N_{\mathrm{b}}=0.20 \AA^{-2}$ are equal to the predicted ion ranges $r$, precisely at the time where $\theta_{c}$ becomes zero. In this way the low-dose behaviour is fully explained by the theory.

By making a graph of $\partial d / \partial N_{b}$ as a function of $\mathrm{d}\left(N_{\mathrm{b}}\right)$ straight lines for $N_{\mathrm{b}}>0.3 \AA^{-2}$ with an intersection $d_{\infty}$ at the axis $\partial d / \partial N_{\mathrm{b}}=0$ are observed. This means that for the high-dose behaviour $d=d_{0}+\left(d_{\infty}-\right.$ $\left.d_{0}\right)\left[1-\exp \left(-A_{\infty} N_{\mathrm{b}}\right)\right]$. The values of $d_{0}$, the initial layer thickness for an amorphous target, are slightly lower than the range $r$, see fig. 5. The values $d_{\infty}$ are also included in fig. 5 and correspond to layer thicknesses in the steady state.

\subsection{Surface roughness}

In order to explain a final layer thickness $d_{\infty}$ much higher than the ion range $r$ and to explain the dilution, a number of model calculations have been carried out [1]. Microscopic surface roughness explains all observed features. By making use of a four-phase system (an amorphous top layer, in which the void fraction $\theta_{v}$ decreases linearly from 1 at $x=0$ to 0 at $x=x_{1}$, is

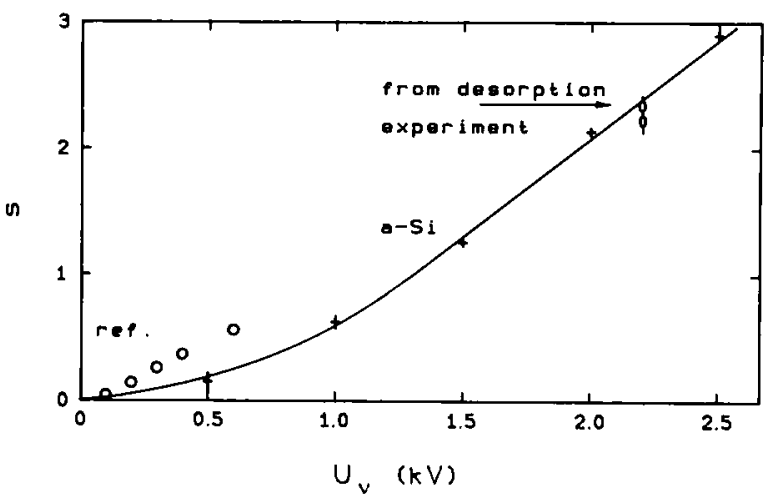

Fig. 6. The calculated sputter coefficients $s$ obtained from ellipsometric results ( + ) as a function of the acceleration voltage. Also included is the calculated value of the desorption experiment. For comparison the sputter coefficients for $\mathrm{Ne}^{+}$on c-Si are included $(\mathrm{O})$ in the graph [7]. positioned on top of an amorphous layer at $x_{1}<x<d$ continued by $\mathrm{c}-\mathrm{Si}$ for $x \geqq d$ ) it can be shown that the standard deviation calculated by making use of the linear regression technique decreases enormously at a certain $x_{1}$. For the $2 \mathrm{keV} \mathrm{Ne}^{+}$ion bombardment at the minimum standard deviation at $x_{1}=51 \AA$ the value $d_{\infty}^{\prime}-x_{1}$ becomes $157 \AA$, which is now comparable with the ion range $r$ of $140 \AA$. Also the deepest pits on the surface act as starting points of penetrating ions. If the total sample surface is called $A_{0}$, then the number of sputtered atoms will be $\mathrm{s} A_{0} \delta N_{\mathrm{b}}$ due to an ion-dose increment $\delta N_{\mathrm{b}}$. The removed number of target atoms will be $1 / 2 A_{0} \delta \Delta d \cdot n_{\text {si }}$, where $1 / 2 A_{0} \delta \Delta d$ denotes the removed volume due to an increase of $\Delta d=d-r$ with $\delta \Delta d$. The balance equation, taking overlap effects into account, becomes:

$\frac{1}{2} A_{0} \delta \Delta d \cdot n_{\mathrm{si}}=s A_{0} \delta N_{\mathrm{b}}\left(1-\frac{\Delta d}{\Delta d_{\infty}}\right)$.

This equation corresponds to the observed behaviour if $A_{\infty}$ is defined by: $A_{\infty}=2 s /\left(n_{\mathrm{si}} \Delta d_{\infty}\right)$. In fig. 6 the calculated values $s=1 / 2 A_{\infty} \Delta d_{\infty} n_{\text {si }}$ are plotted as a function of the acceleration voltage. Also included is the sputter coefficient for a $2.2 \mathrm{kV} \mathrm{Ne}^{+}$bombardment which explains the collected gas at desorption experiments [1]. The sputter coefficients for $\mathrm{Ne}^{+}$on $\mathrm{Si}[7]$ are included as a reference.

\section{References}

[1] A.H.M. Holtslag, Noble-gas ion bombardment on clean silicon surfaces, studied using ellipsometry and desorption, Thesis, Twente University of Technology (1986).

[2] F.F. Morehead and B.L. Crowder, Radiat. Eff. 6 (1979) 27.

[3] F. Abeles, Optical properties of solids (North-Holland, Amsterdam, 1972) p. 277.

[4] E.A. Davis and N.F. Mott, Phil. Mag. 22 (1970) 903.

[5] G.D. Cody, B.G. Brooks and B. Abeles, Solar Energy Mater: 8 (1982) 231.

[6] J.F. Ziegler, J.P. Biersack and U. Littmark, The stopping and range of ions in solids, vol. 1 (Pergamon, New York, 1985).

[7] R. Behrisch, Sputtering by particle bombardment I, Topics in Applied Physics vol. 47 (Springer, Berlin, 1981) p. 169. 